\title{
SOLVING BELTRAMI EQUATIONS BY CIRCLE PACKING
}

\author{
ZHENG-XU HE
}

\begin{abstract}
We use Andreev-Thurston's theorem on the existence of circle packings to construct approximating solutions to the Beltrami equations on Riemann surfaces. The convergence of the approximating solutions on compact subsets will be shown. This gives a constructive proof of the existence theorem for Beltrami equations.
\end{abstract}

\section{INTRODUCTION}

A circle packing in the plane or in a closed Riemannian 2-manifold of constant curvature is a collection of closed round disks with disjoint interiors. The nerve of such circle packing is the embedded graph whose vertices are the centers of the disks and whose edges are the shortest geodesic arcs joining the centers of each pair of intersecting disks. By Andreev-Thurston's theorem, for any triangulation of the Riemann sphere $S^{2}$, there is a circle packing on $S^{2}$, unique up to a Möbius transformation, whose nerve is isotopic to the 1-skeleton of the triangulation (see [T, Corollary 13.6.2, or MR]). Based on this fact, William P. Thurston suggested a geometric approach to the Riemann mapping theorem. Recently, Burt Rodin and Dennis Sullivan [RS] carried out this approach and verified the convergence of Thurston's scheme. In this paper, we will use this circle packing method to construct approximations to solutions $f: \Omega \rightarrow \mathbf{C}$ of the Beltrami equation:

$$
\partial_{\bar{z}} f(z)=\lambda(z) \partial_{z} f(z) \text { a.e. } z=x+i y \in \Omega,
$$

where $\Omega$ is some open Jordan domain in $\mathbf{C}$, and $\lambda: \Omega \rightarrow \mathbf{C}$ is some measurable function with

$$
\|\lambda\|_{\infty}=\underset{z \in \Omega}{\operatorname{ess} \sup }|\lambda(z)|<1 .
$$

$(\lambda(z)$ is called the complex dilatation of $f(z)$ if $f$ is a quasiconformal mapping satisfying (1.1) (see [A or LV]).)

Received by the editors August 15, 1988 and, in revised form, December 22, 1988.

1980 Mathematics Subject Classification (1985 Revision). Primary 30C60; Secondary 65E05, $57 \mathrm{~N} 05$.

Key words and phrases. Circle packing, quasiconformal mapping, Beltrami equation, PL complex.

Supported by the DARPA Applied and Computational Mathematics Program, No. 86-A227500. 
Our methods can be generalized to construct approximations to solutions of Beltrami equations on an arbitrary Riemann surface. Let $\Omega$ be an open domain in a closed Riemann surface $M$ such that $\partial \Omega$ is a finite union of Jordan curves, and let $\lambda: \Omega \rightarrow \mathbf{C}$ be a (Lebesgue) measurable function with $\|\lambda\|_{\infty}<1$. In this case, there is a closed Riemann surface $N$, a finite union $R$ of disjoint closed round disks in $N$, and a quasiconformal homeomorphism $f: \Omega \rightarrow N \backslash R$ with complex dilatation $\lambda$. Letting $\lambda=0$, this implies a generalization of the Riemann Mapping Theorem: $\Omega$ is conformally homeomorphic to a domain in a closed Riemannian 2-manifold of constant curvature, whose boundary is a finite union of round circles.

In a forthcoming paper [H], we will give an estimate on the constants $s_{m}$ in the Hexagonal Packing Lemma of [RS, §4]. In this way, our proof is in fact constructive. We refer to [RS, Appendix 2] for an algorithm (after Thurston) for finding a circle packing with a prescribed pattern.

The existence of local solutions to the Beltrami equation for a Hölder continuous $\mu$ is classical (see $[\mathrm{K}, \mathrm{Li}, \mathrm{Be}$, or $\mathrm{C}]$ ), and the global existence can be obtained using the uniformization theorem for Riemann surfaces. The existence theorem for a measurable $\mu$ was first obtained by Morrey [Mo] by solving the boundary value problem for the real part of the solution (see also [Ma]), and then by Bers and Nirenberg [BN] using the representation theory for linear elliptic systems (see the Bibliographical Note in $[A B$, p. 385]). Later, Boyarksii [Bo] and Ahlfors and Bers [AB] obtained an important formula for the global solution in the entire plane based on some integral transformations. In [LV], following an idea of [La], an approximating solution is constructed by sewing together the solutions on small rectangles (which exist since we may assume $\lambda$ is constant there). This requires repeated application of the Riemann Mapping Theorem. The proof presented in this paper differs from the others in that it is purely geometric and can be implemented on a computer.

The author is very much indebted to his advisor, Michael H. Freedman, for his constant encouragement and many helpful suggestions, and to the referee for valuable comments.

\section{TERMINOLOGY AND NOTATIONAL CONVENTIONS}

Let $X$ be a topological space. By a $P L$ complex on $X$, we will mean a pair $(G, g)$ consisting of a simplicial complex $G$ and a homeomorphism $g:|G| \rightarrow X$. If $X^{\prime}$ is a subspace of $X$ such that $X^{\prime}=g\left(\left|G^{\prime}\right|\right)$ for some subcomplex $G^{\prime}$ of $G$, then $\left(G^{\prime}, g^{\prime}=g /\left|G^{\prime}\right|\right)$ is a $P L$ complex on $X^{\prime}$, and we denote $(G, g) / X^{\prime}=\left(G^{\prime}, g^{\prime}\right)$. Let $\sigma$ be an $n$-simplex of the simplicial complex $G$, we call $g(\sigma)$ an $n$-simplex of the $P L$ complex $(G, g)$. Two $P L$ complexes $(G, g)$ and $(H, h)$ on $X$ are equivalent if there is a simplicial homeomorphism $\phi:|G| \rightarrow|H|$ such that $g=h \circ \phi$.

Let $X$ be any polyhedron in some finite dimensional real linear space $V$. A $P L$ complex $(G, g)$ on $X$ is called an affine triangulation on $X$ if for any 
simplex $\sigma$ of $G$, the map $g / \sigma: \sigma \rightarrow X \subset V$ is the restriction of some affine map from the affine subspace spanned by $\sigma$ to $V$.

Suppose $X$ is an open or closed domain in some closed 2-manifold $M$ with a conformal structure (= complex structure), and let $(G, g)$ be a $P L$ complex on $X$. Then $|G|$ is an oriented 2-manifold whose orientation is chosen so that $g$ is sense preserving. We will call $(G, g)$ a $K$-quasiconformal PL complex $(K \geq 1)$, if for any triangle (= 2-simplex) $\sigma$ of $G, g \circ \phi: \sigma^{\prime} \rightarrow X \subset M$ is $K$-quasiconformal onto its image, where $\sigma^{\prime}$ is some equilateral triangle in $\mathbf{C}$ (with the standard conformal structure) and $\phi: \sigma^{\prime} \rightarrow \sigma$ is some sense preserving affine homeomorphism. Note that if $\left(G, g_{1}\right)$ and $\left(G, g_{2}\right)$ are, respectively, $K_{1}$ and $K_{2}$-quasiconformal $P L$ complexes on $X_{1}$ and $X_{2}$, then $g_{2} \circ g_{1}^{-1}: X_{1} \rightarrow X_{2}$ is a $K_{1} K_{2}$-quasiconformal homeomorphism.

\section{CONSTRUCTION OF APPROXIMATING SOLUTIONS}

Let $\Omega$ be an open Jordan domain in $\mathrm{C}$. Let $\delta>0$ be any small number. Construct a brick decomposition of $\mathbf{C}$ as in Figure 1, such that each brick is a parallel translation of $\{x=i y ; 0 \leq x \leq 2 \delta, 0 \leq y \leq \sqrt{3} \delta / 2\}$. Any two intersecting bricks intersect in a line segment of length $\delta$ or $\sqrt{3} \delta / 2$. Let $\bar{\Omega}_{\delta}$ be the minimal Jordan domain containing all bricks which intersect with $\bar{\Omega}$. Then $\bar{\Omega}_{\delta}$ is a closed polyhedral Jordan domain, which can be decomposed as the union of a family of (closed) rectangles of side length $\delta$ and $\sqrt{3} \delta / 2$, such that any two intersecting rectangles intersect in a common vertex or in a common edge. (In the following, a rectangle in $\bar{\Omega}_{\delta}$ will mean a rectangle in this family.) It is clear that $\bar{\Omega} \subset \bar{\Omega}_{\delta}$ and $\bar{\Omega}_{\delta}$ converges to $\bar{\Omega}$ when $\delta \rightarrow 0$ in the sense that any compact subset of $\widehat{\mathbf{C}} \backslash \bar{\Omega}$ is contained in $\widehat{\mathbf{C}} \backslash \bar{\Omega}_{\delta}$ for $\delta$ small enough.

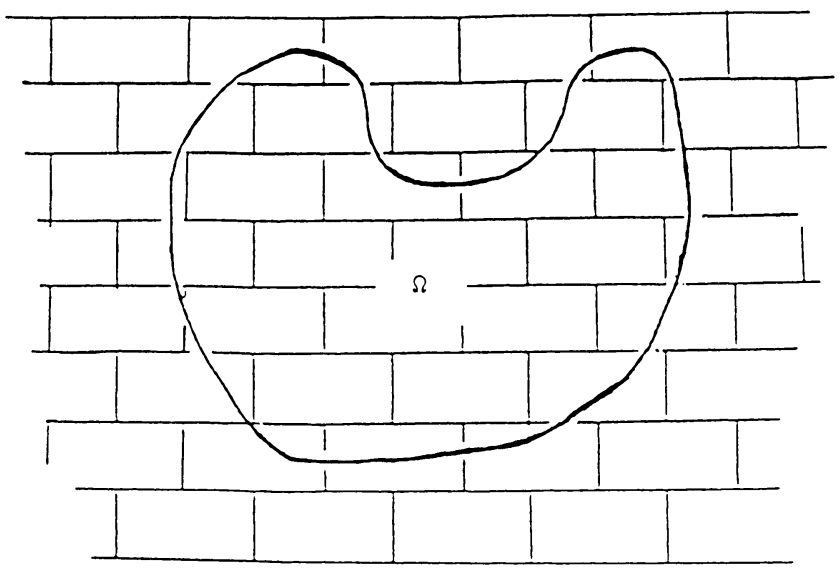

FIGURE 1 
Let $\lambda: \Omega \rightarrow \mathbf{C}$ be a measurable function with $\|\lambda\|_{\infty}<1$. For any rectangle $Q$ in $\bar{\Omega}_{\delta}$, we let

$$
\lambda(Q)= \begin{cases}\frac{1}{A(Q)} \iint_{Q} \lambda(x+i y) d x d y, & \text { if } Q \subset \Omega, \\ 0, & \text { otherwise, }\end{cases}
$$

where $A(\cdot)$ denotes the area. Define approximations $\lambda_{\delta}: \Omega \rightarrow \mathbf{C}$ of $\lambda$ by

$$
\lambda_{\delta}(z)=\lambda(Q) \text { a.e. } z \in Q \cap \Omega, \forall Q .
$$

Then,

$$
\lim _{\delta \backslash 0} \lambda_{\delta}(z)=\lambda(z) \text { a.e. } z \in \Omega \text {. }
$$

For any triangle $\Delta$ in the plane $\mathbf{C}$ there is some orientation-preserving (real) affine self-mapping $A$ of $\mathbf{C}$, which maps $\Delta$ onto some equilateral triangle. The complex dilatation of $A$ (which is constant throughout $\mathbf{C}$ ) depends only on the triangle $\Delta$. Let us call it the complex dilatation of the triangle $\Delta$.

The approximating solution to the Beltrami equation will be realized by some straightening map from some affine triangulation $\left(T_{\delta}, t_{\delta}\right)$ for $\bar{\Omega}_{\delta}$ to the affine triangulation determined by the associated circle packing in the unit disk $D$. In brief, one may assume $\lambda$ is constant $(=\lambda(Q))$ on each rectangle $Q$, pack these rectangles with pull-backs of the regular hex-packs of very fine mesh under appropriate affine maps, and observe from the conformal packing theory that, several generations inside the hexagonal triangulations, the complex dilatation of the straightening map is approximately $\lambda$. To make these packs fit together on common boundaries of rectangles, we will need some construction near the boundaries of each rectangle. And, in order to apply the Leng-Area Lemma of [RS], we will pack the boundary rectangles (i.e., rectangles $Q$ with $Q \cap$ $\left.\partial \bar{\Omega}_{\delta} \neq \phi\right)$ in such a way that any vertex is the end-point of at most six 1simplexes. These boundary regions have small areas, and allow us to choose arbitrary quasiconformal triangulation there.

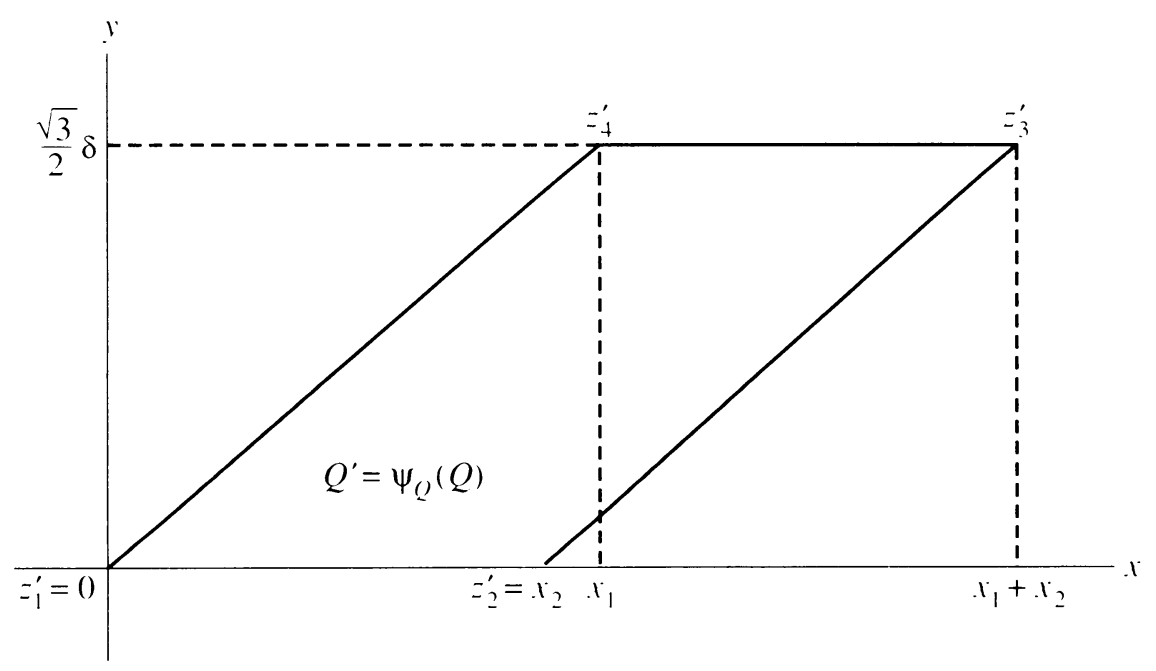

FIGURE 2 
So let $Q$ be rectangle in $\bar{\Omega}_{\delta}$ with $Q \cap \partial \bar{\Omega}_{\delta}=\phi$. Denote by $z_{1}, z_{2}, z_{3}$, and $z_{4}$ its lower left, lower right, upper right, and upper left vertices, respectively. Let $\psi_{Q}: \mathbf{C} \rightarrow \mathbf{C}$ be the following map:

$$
\psi_{Q}(z)=\frac{1+\overline{\lambda(Q)}}{1-|\lambda(Q)|^{2}}\left(z-z_{1}+\lambda(Q)\left(\bar{z}-\bar{z}_{1}\right)\right) .
$$

$\psi_{Q}$ is an affine conformal homeomorphism with complex dilatation $\lambda(Q)$, and maps $Q$ onto the parallelogram $Q^{\prime}$ with vertices $z_{1}^{\prime}=\psi_{Q}\left(z_{1}\right)=0, z_{2}^{\prime}=$ $\psi_{Q}\left(z_{2}\right)=x_{2}, \quad z_{3}^{\prime}=\psi_{Q}\left(z_{3}\right)=x_{1}+x_{2}+(\sqrt{3} \delta / 2) i$, and $z_{4}^{\prime}=\psi_{Q}\left(z_{4}\right)=z_{1}+$ $(\sqrt{3} \delta / 2) i$, where

$$
x_{1}=\frac{\sqrt{3} \operatorname{Im} \lambda(Q)}{1-|\lambda(Q)|^{2}} \delta, \quad x_{2}=\frac{|1+\lambda(Q)|^{2}}{1-|\lambda(Q)|^{2}} \delta
$$

(see Figure 2).

Let $n=2[1 / 2 \delta]$, and let $H_{\delta}$ be the simplicial complex whose underlying space is $\mathbf{C}$ such that:

(i) each 1-simplex of $H_{\delta}$ has length $\delta / n$;

(ii) the origin of $\mathbf{C}$ is a vertex of $H_{\delta}$; and

(iii) the real line in $\mathrm{C}$ is the underlying space of some subcomplex of $H_{\delta}$. Then the 1-skeleton of $H_{\delta}$ is the nerve of some regular hexagonal circle packing $P_{\delta}$ of $\mathrm{C}$ formed by closed disk of radius $\delta / 2 n$ (see Figure 3 ).

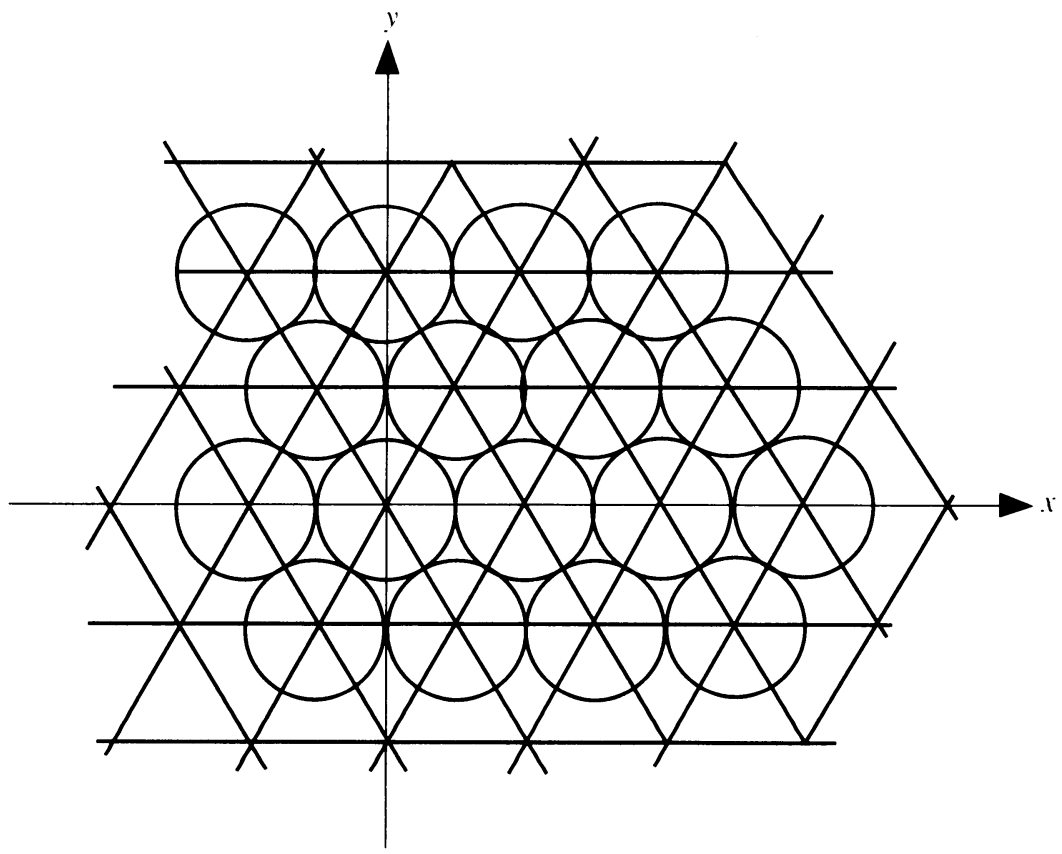

FIGURE 3 


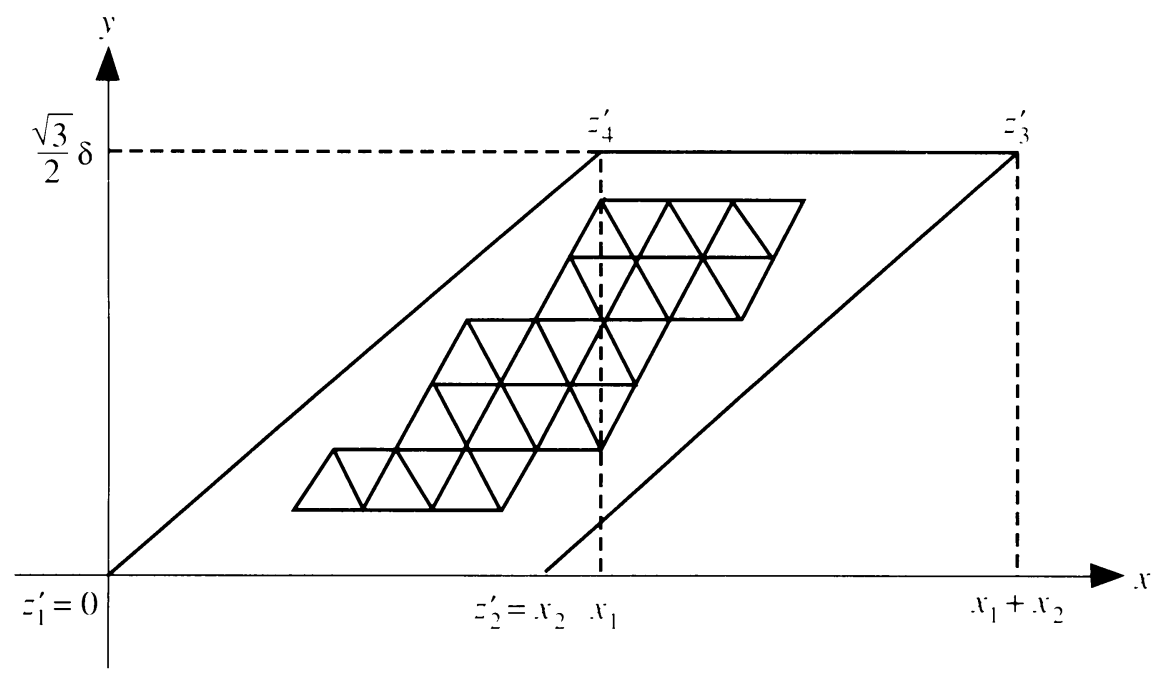

FIGURE 4

Let $H\left(Q^{\prime}\right)$ be the collection of all triangles of $H_{\delta}$ lying in $Q^{\prime}$ and having distance at least $\delta / 2 n$ from $\partial Q^{\prime}$, together with their edges and vertices. Then $H\left(Q^{\prime}\right)$ is a simplicial complex and $\left|H\left(Q^{\prime}\right)\right|$ is some polyhedral Jordan domain in $Q^{\prime}$ (see Figure 4).

In the following throughout the paper, $c$ and $C$ will denote some constants depending only on $\|\lambda\|_{\infty}$ with $0<c \leq 1, C \geq 1$.

By (3.5) and the fact that $|\lambda(Q)| \leq\|\lambda\|_{\infty}$, the slope of the side $z_{1}^{\prime} z_{4}^{\prime}$ (or $z_{2}^{\prime} z_{3}^{\prime}$ ) of the parallelogram $Q^{\prime}$ is bounded from below, and its length is between $c \delta$ and $C \delta$. As the triangles in $H\left(Q^{\prime}\right)$ have length $\delta / n$, there is a triangulation $\left(T\left(Q^{\prime}\right), t^{\prime}\right)$ on $Q^{\prime}$, which agrees with the affine triangulation $\left(H\left(Q^{\prime}\right)\right.$, id $)$ on $Q_{i}^{\prime}=\left|H\left(Q^{\prime}\right)\right|$, whose restriction on each side of the parallelogram $Q^{\prime}$ is equal to the affine triangulation (of the side) consisting of $n$ 1-simplexes of equal lengths, and whose 1 -simplexes have lengths between $c \delta / n$ and $C \delta / n$. To construct this triangulation, let $z_{j}^{\prime \prime}$ be the vertex of $H\left(Q^{\prime}\right)$ nearest to $z_{j}^{\prime}, j=1,2,3,4$. Then the arcs $z_{j}^{\prime} z_{j}^{\prime \prime}$ divide the annulus $Q^{\prime} \backslash\left|H\left(Q^{\prime}\right)\right|$ into four parts: $R_{12}=$ the trapezoid $z_{1}^{\prime} z_{2}^{\prime} z_{2}^{\prime \prime} z_{1}^{\prime \prime}, R_{23}=$ the portion between $z_{2}^{\prime} z_{2}^{\prime \prime}$ and $z_{3}^{\prime} z_{3}^{\prime \prime}, R_{34}=$ the trapezoid $z_{3}^{\prime} z_{4}^{\prime} z_{4}^{\prime \prime} z_{3}^{\prime}$, and $R_{41}$ (see Figure 5).

The boundaries of these four regions are already triangulated by letting $z_{j}^{\prime} z_{j}^{\prime \prime}$ be 1-simplex, and our task is to extend these affine triangulations from boundaries to the interiors. This is easily done for $R_{12}$ (and similarly for $R_{34}$ ): first connect (by adding a 1-simplex) any vertex on $z_{1}^{\prime} z_{2}^{\prime}$ with the nearest vertex on $z_{1}^{\prime \prime} z_{2}^{\prime \prime}$, and connect any vertex on $z_{1}^{\prime \prime} z_{2}^{\prime \prime}$ with the nearest vertex on $z_{1}^{\prime} z_{2}^{\prime}$; these arcs divide $E_{12}$ into triangles and/or trapezoids whose sides are all 1simplexes; then for each of these trapezoids take any one of the two diagonals to be 1 -simplex. For the region $R_{41}$, let $w$ be a vertex on $z_{1}^{\prime} z_{4}^{\prime}$ different from 


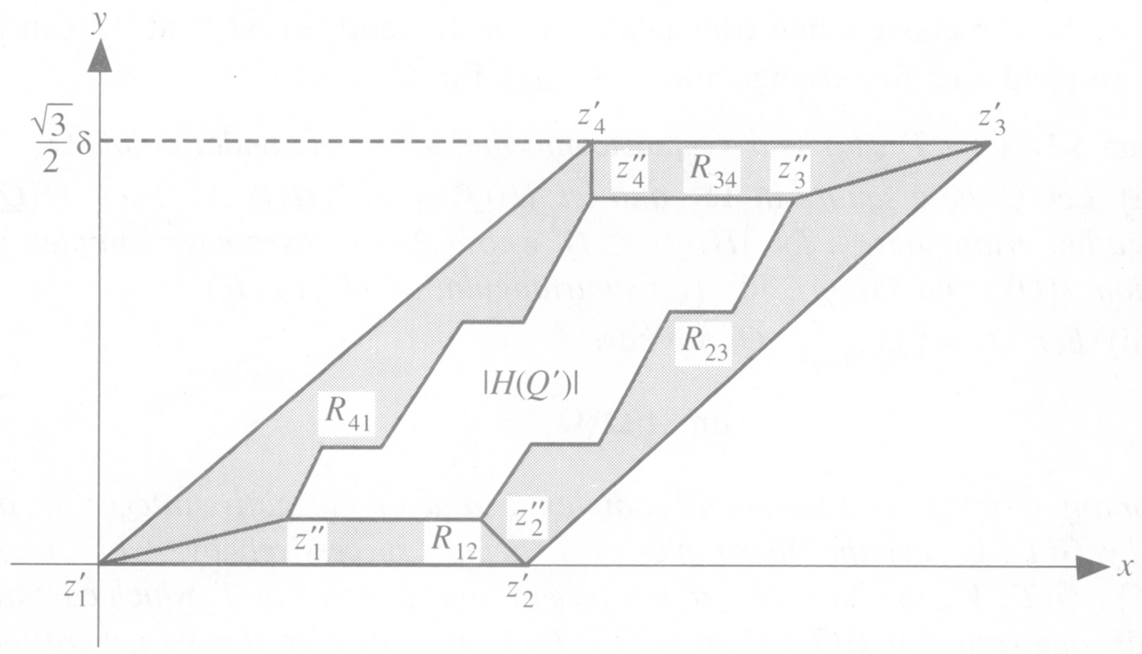

Figure 5

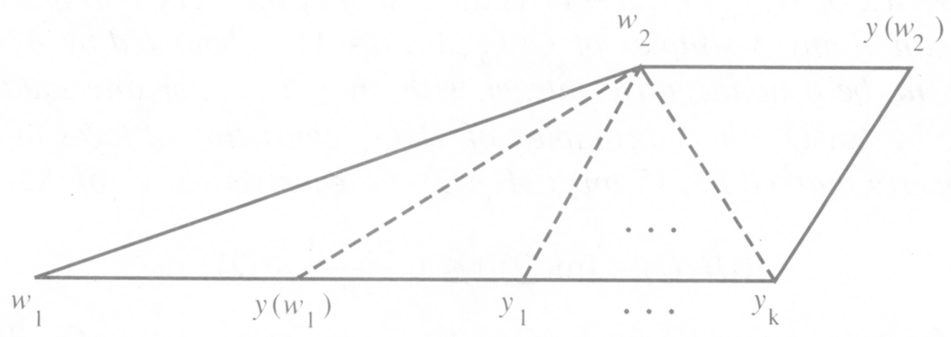

Figure 6

$z_{1}^{\prime}$ and $z_{4}^{\prime}$. The imaginary part $\operatorname{Im}(w)$ of $w$ is a multiple of $\sqrt{3 \delta} / 2 n$, so there are vertices of $H\left(Q^{\prime}\right)$ whose imaginary part equals $\operatorname{Im}(w)$. Let $y(w)$ be the one which is nearest to $w$, and connected each $w$ with $y(w)$. These arcs divide $R_{41}$ into two triangles and $n-1$ trapezoids of the form shown in Figure 6 , where $y_{j}$ 's may not be present. Finally by connecting $y\left(w_{1}\right)$ and each $y_{j}$ with $w_{2}$, we get an affine triangulation for $R_{41}$. Analogous construction could be done for $R_{23}$. Glueing these affine triangulations, we obtain the desired $\left(T\left(Q^{\prime}\right), t^{\prime}\right)$.

Let $(T(Q), t)=\left(T\left(Q^{\prime}\right),\left(\psi_{Q}\right)^{-1} \circ t^{\prime}\right)$. Then $(T(Q), t)$ is a $C$-quasiconformal affine triangulation on the rectangle $Q$, and its restriction on each side of $Q$ is the affine triangulation with $n 1$-simplexes of equal lengths. So these affine triangulations can be glued together to form a $C$-quasiconformal affine triangulation for $\bar{\Omega}_{\delta}=\bigcup_{Q \cap \partial \bar{\Omega}_{\delta}=\phi} Q$. On the other hand, by intersecting the region $\bar{\Omega}_{\delta} \backslash$ int $\widetilde{\Omega}_{\delta}=\bigcup_{Q \cap \partial \widetilde{\Omega}_{\delta} \neq \phi} Q$ the regular hexagonal triangulations $H_{\delta} / n$ of the plane whose triangles have side length $\delta / n$, we get an affine triangulation for $\bar{\Omega}_{\delta} /$ int $\widetilde{\Omega}_{\delta}$. Since $n$ is even, each horizontal 1-simplex of $Q \cap H_{\delta} / n$ has length 
$\sqrt{3} \delta / 2$. So the above affine triangulations for $\widetilde{\Omega}_{\delta}$ and for $\bar{\Omega}_{\delta} \backslash$ int $\widetilde{\Omega}_{\delta}$ can be glued to yield an affine triangulation $\left(T_{\delta}, t_{\delta}\right)$ for $\bar{\Omega}_{\delta}$.

Lemma 3.1. (i) $\left(T_{\delta}, t_{\delta}\right)$ is a C-quasiconformal affine triangulation on $\bar{\Omega}_{\delta}$.

(ii) Let $Q$ be a square in $\widetilde{\Omega}_{\delta}$ and let $H(Q)=\psi_{Q}^{-1}\left(H\left(Q^{\prime}\right)\right)$. Then $\left.H(Q)\right)$ is an affine triangulation for $|H(Q)| \subset Q$ whose 2-simplexes have complex dilatation $\lambda(Q)$, and $(H(Q), \mathrm{id})$ is a subtriangulation of $\left(T_{\delta}, t_{\delta}\right)$.

(iii) For $\widetilde{\Omega}_{\delta}=\bigcup_{Q \cap \partial \bar{\Omega}_{\delta}=\phi} Q$, we have

$$
\lim _{\delta \backslash 0} A\left(\Omega \backslash \tilde{\Omega}_{\delta}\right)=0 .
$$

For any vertex $V$ in a simplicial complex $T$ and a nonnegative integer $m$, denote by $G(T, V, m)$ the subcomplex of $T$ defined successively by: $G(T, V, 0)$ $=\{V\}, G(T, V, m+1)=\{\sigma ; \sigma$ is a face of some simplex of $T$ which contains at least one vertex of $G(T, V, m)\} . G(T, v, m)$ is called the $m$ generations about the vertex $V$ in the complex $T$. Then we have:

(iv) For any vertex $V$ of $T_{\delta}$ lying on the boundary $\partial\left|T_{\delta}\right|$ of the 2-manifold $\left|T_{\delta}\right|$, each vertex of $G\left(T_{\delta}, V, n-1\right)$ is the end of at most six 1-simplexes of $T_{\delta}$ and the length of any 1-simplex of $G\left(T_{\delta}, V, n-1\right)$ is bounded by $\delta / n$.

(v) Let $m$ be a nonnegative integer with $m \leq \mathrm{cn}$. For any square $Q$ in $\widetilde{\Omega}_{\delta}$, denote by $\operatorname{Im}(Q)$ the subcomplex of $H(Q)$ consisting of faces of triangles $\Delta$ with property that $G\left(T_{\delta}, V, m\right) \subset H_{n}(Q)$ for each vertex $V$ of $\Delta$. Then

$$
A(|H(Q)| \backslash|\operatorname{Im}(Q)|) \leq C \frac{m+1}{n} A(Q) .
$$

Note that for any vertex $V$ in $I_{m}(Q), G\left(T_{\delta}, V, m\right)=G\left(H_{n}(Q), V, m\right)$ is abstractly isomorphic to the $m$ generators about a vertex in a regular hexagonal triangulation on the plane.

Proof. (i) Follow directly from the construction. (ii) It is obvious since all triangles of $H\left(Q^{\prime}\right)$ are equilateral and $\psi_{Q}$ is affine and has complex dilatation $\lambda(Q)$. (iii) Any compact subset of $\Omega$ is contained in $\widetilde{\Omega}_{\delta}$ for $\delta$ small, this implies (3.6). (iv) We have $\bar{\Omega}_{\delta} \backslash$ int $\widetilde{\Omega}_{\delta}=\bigcup_{Q \cap \partial \bar{\Omega}_{\delta} \neq \phi} Q$, where each $Q$ is a rectangle of side lengths $\delta$ and $\sqrt{3} \delta / 2$, and the triangulation $T_{\delta} / \bar{\Omega}_{\delta} \backslash$ int $\widetilde{\Omega}_{\delta}$ is obtained by intersecting $\bar{\Omega}_{\delta} \backslash$ int $\widetilde{\Omega}_{\delta}$ with the regular hexagonal triangulation of mesh $\delta / n$, the statement follows. (v) It is clear that the distance from any point on $\partial\left|H\left(Q^{\prime}\right)\right|$ to $\partial Q^{\prime}$ is bounded by $\sqrt{3} \delta / 2 n+\delta / n$, and hence the distance from any point on $\partial\left|I_{m}\left(Q^{\prime}\right)\right|$ (where $I_{m}\left(Q^{\prime}\right)=\psi_{Q}\left(I_{m}(Q)\right)$ ) to $\partial Q^{\prime}$ is bounded by $\sqrt{3} \delta / 2 n+\delta / n+m \delta / n$. But $\psi_{Q}: Q \rightarrow Q^{\prime}$ is bilipschitz, so the distance from $\partial\left|I_{m}(Q)\right|$ to $\partial Q$ is bounded by $C(m+1) \delta / n$, which implies (3.7).

Since $\left|T_{\delta}\right|$ is a closed p.l. 2-dimensional disk, there is a circle packing $P_{\delta}$ in the closed unit disk $\bar{D}$ whose nerve is isotopic to the 1-skeleton of $T_{\delta}$, so that each vertex of $T_{\delta}$ lying on $\partial\left|T_{\delta}\right|$ corresponds to a closed disk of $P_{\delta}$ tangent to the unit circle $\partial D$. Furthermore, $P_{\delta}$ is unique up to a Möbius transformation 
of the unit disk. To see this, it is enough to consider the simplicial complex $T_{\delta} \cup\left(\left(T_{\delta} / \partial\left|T_{\delta}\right|\right) * V\right)$, where $V$ is an added vertex and "* " denotes the join operation, and then apply Andreev-Thurston's theorem stated in the introduction (see also [RS]).

Let $g_{\delta}:\left|T_{\delta}\right| \rightarrow \bar{D}$ be the map which sends any vertex of $T_{\delta}$ to the center of the corresponding disk and then extends it linearly on each simplex of $T_{\delta}$. Then $g_{\delta}$ is a sense preserving homeomorphisms of $\left|T_{\delta}\right|$ onto its image $\bar{D}_{\delta}=$ $g_{\delta}\left(\left|T_{\delta}\right|\right) \subset \bar{D}$. By composing with a Möbius transformation of the unit disk, we may arrange $g_{\delta}$ to satisfy

$$
g_{\delta} \circ t_{\delta}^{-1}\left(z_{0}\right)=0, \quad g_{\delta} \circ t_{\delta}^{-1}\left(z_{0}^{\prime}\right) \in(0,1) \subset \mathbf{R}
$$

for two prescribed points $z_{0}, z_{0}^{\prime} \in \Omega$.

Now define $f_{\delta}: \Omega \rightarrow D=\operatorname{int} \bar{D}$ to be the restriction of $g_{\delta} \circ t_{\delta}^{-1}$ on $\Omega$. Then we have

$$
f_{\delta}\left(z_{0}\right)=0, \quad f_{\delta}\left(z_{0}^{\prime}\right) \in(0,1) .
$$

We will show in the next section that for $\delta \rightarrow 0, f_{\delta}$ converges to some solution of Beltrami equation.

\section{Convergence of approximating solutions}

In this section, we prove the following convergence theorem.

Theorem 4.1. Let $\lambda: \Omega \rightarrow \mathbf{C}$ be a measurable function with $\|\lambda\|_{\infty}<1$. For any sequence $\delta_{k}, \delta_{k}>0$, with $\delta_{k} \rightarrow 0$, let $f_{\delta_{k}}: \Omega \rightarrow D$ be the maps constructed in $\S 3$. Then $f_{\delta_{k}}$ has a subsequence converging uniformly on every compact subset of $\Omega$ to some quasiconformal homeomorphism $f: \Omega \rightarrow D$ which solves Beltrami equation:

$$
\frac{\partial_{\bar{z}} f(z)}{\partial_{z} f(z)}=\lambda(z) \text { a.e. } z \in \Omega .
$$

Since the solution of Beltrami equation is unique up to a conformal homeomorphism (see [LV, IV.5.3]), the solution $f: \Omega \rightarrow D$ is unique if we require

$$
f\left(z_{0}\right)=0, \quad f\left(z_{0}^{\prime}\right) \in(0,1) .
$$

It follows that (see also (3.9)):

Theorem 4.2. Let $\delta \rightarrow 0$, the maps $f_{\delta}$ constructed in $\S 3$ converge uniformly on every compact subset to the solution $f: \Omega \rightarrow D$ of (1.1) and (4.1).

In [H], we will show that $f_{\delta}$ converges globally uniformly to $f$. The proof requires an analysis of the boundaries of $\bar{\Omega}_{\delta}$ and $\bar{\Omega}_{\delta}=g_{\delta}(|T|)$, and uses an improved version of the convergence theorem for quasiconformal mapping.

Proof of Theorem 4.1. By Lemma 3.1(i), any vertex of $T_{\delta}$ is the end of at most $C$ 1-simplexes of $T_{\delta}$. Hence, any disk of $P_{\delta}$ is tangent to at most $C$ other disks. Then the Ring Lemma of [RS] (and its proof) shows that any 
two neighboring disks of the circle packing $P_{\delta}$ have radii with ratio bounded by $C$. It follows that $\left(T_{\delta}, g_{\delta}\right)$ is a $C$-quasiconformal $P L$ complex on $\bar{\Omega}_{\delta}=$ $g_{\delta}(|T|)$. This fact and Lemma 4.1(i) implies that $g_{\delta} \circ t_{\delta}^{-1}: \bar{\Omega}_{\delta} \subset \bar{\Omega}$ is a $C$ quasiconformal homeomorphism.

Let $\delta_{k} \rightarrow 0$, and let $f_{\delta_{k}}^{\prime}=g_{\delta_{k}} \circ t_{\delta}^{-1}$. Then $f_{\delta_{k}}=f_{\delta_{k}}^{\prime} / \Omega: \Omega \rightarrow D$ is $C$ quasiconformal, as $f_{\delta_{k}}^{\prime}$ is. By the convergence theorem for quasiconformal maps (see, e.g., [LV, Theorems II.5.1 and 5.3]), there is a subsequence of $f_{\delta_{k}}$, still denoted by $f_{\delta_{k}}$, which converges uniformly on every compact subset of $\Omega$ to some map $f: \Omega \rightarrow D$, and $f$ is either a constant or quasiconformal homeomorphism onto its image. By Lemma 3.1(iv) and the Length-Area Lemma of [RS, §4], the radii of boundary circles of $P_{\delta_{k}}$ converge to 0 as $\delta_{k} \rightarrow 0$. It follows that $f_{\delta_{k}}\left(\Omega_{\delta_{k}}\right)$ converges to $D$. So $f$ is a quasiconformal map onto $D$.

It remains to show that $f$ has complex dilatation $\lambda$. Let $\mu_{k}: \Omega \rightarrow \mathbf{C}$ be the complex dilatation of the quasiconformal map $f_{\delta_{k}}$, i.e.,

$$
\partial_{\xi} f_{\delta_{k}}(z)=\mu_{k}(z) \cdot \partial_{z} f_{\partial_{k}}(z) \text { a.e. } z \in \Omega \text {. }
$$

By the Convergence Theorem of Quasiconformal Mappings (see, e.g., [LV, Theorem IV.5.2]), it suffices to show

$$
\lim _{k \rightarrow \infty} \mu_{k}(z)=\lambda(z) \quad \text { a.e. } z \in \Omega \text {. }
$$

Thus, the proof of Theorem 4.1 is complete if (4.3) is verified. Let $m_{k}$ be a sequence of positive integers such that $m_{k} \rightarrow \infty$ and $m_{k} / n_{k} \rightarrow 0$ (recall that $\left.n_{k}=2\left[1 / 2 \delta_{k}\right]\right)$. By Lemma $3.1(\mathrm{v})$, for any rectangle $Q$ in $\widetilde{\Omega}_{\delta}$ and any vertex $V$ in $I_{m_{k}}(Q)$, the $m_{k}$ generations in the circle packing $P_{\delta_{k}, m_{k}}$ about the disk corresponding to $V$ is combinatorially equivalent to $m_{k}$ generations of regular hexagonal packing about one of its disk. So, by the Hexagonal Packing Lemma of [RS, §4], the ratio of lengths of any two edges in any triangle of $g_{\delta_{k}} \circ t_{\delta_{k}}^{-1}\left(I_{m_{k}}(Q)\right)$ is less than $1+s_{m_{k}}$, for some sequence $s_{m}$, decreasing to zero. Therefore $g_{\delta_{k}} / \sigma: \sigma \rightarrow D$ is $1+s_{m_{k}}^{\prime}$-quasiconformal for any (equilateral) triangle $\sigma$ of $t_{\delta_{k}}^{-1}\left(I_{m_{k}}(Q)\right)$, where $s_{m_{k}}^{\prime} \rightarrow 0$. Using Lemma 3.1(ii), (3.2), and (4.2), it follows that

$$
\left|\mu_{k}(z)-\lambda_{\delta_{k}}(z)\right| \leq \varepsilon_{k} \quad \text { a.e. } z \in\left|I_{m_{k}}(Q)\right|, \forall Q \subset \widetilde{Q}_{\delta} .
$$

Let

$$
I_{k}=\bigcup_{Q \cap i \bar{\Omega}_{j}=\phi}\left|I_{m_{k}}(Q)\right| .
$$

They by (3.7) and the fact $m_{k} / n_{k} \rightarrow 0$, we have

$$
\lim _{k \rightarrow 0} \frac{A\left(\widetilde{\Omega}_{\delta_{k}}-I_{k}\right)}{A\left(\widetilde{\Omega}_{\delta_{k}}\right)}=0 .
$$


In light of (3.6), this implies that

$$
\lim _{k \rightarrow \infty} A\left(\Omega \backslash I_{k}\right)=0 .
$$

Using (4.4), we get

$$
\begin{aligned}
\iint_{\Omega}\left|\mu_{k}(z)-\lambda_{\delta_{k}}(z)\right| d x d y= & \iint_{\Omega \backslash I_{k}}\left|\mu_{k}(z)-\lambda_{\delta_{k}}(z)\right| d x d y \\
& +\iint_{I_{k}}\left|\mu_{k}(z)-\lambda_{\delta_{k}}(z)\right| d x d y \\
\leq & 2 A\left(\Omega \backslash I_{k}\right)+\varepsilon_{k} A\left(\left|I_{k}\right|\right) .
\end{aligned}
$$

By (4.6) and the fact that $\varepsilon_{k} \rightarrow 0$, we obtain

$$
\lim _{k \rightarrow \infty} \iint_{\Omega}\left|\mu_{k}(z)-\lambda_{\delta_{k}}(z)\right| d x d y=0 .
$$

So by extracting a subsequence, we have

$$
\lim _{k \rightarrow \infty}\left(\mu_{k}(z)-\lambda_{\delta_{k}}(z)\right)=0 \quad \text { a.e. } z \in \Omega
$$

This together with (3.3) yields (4.3), and hence $f$ solves the Beltrami equation.

\section{BELTRAMI EQUATIONS IN RIEMANN SURFACES}

Let $\Omega$ be an open domain in a closed Riemann surface $M$, such that $\partial \Omega$ is a finite union of Jordan curves (= topologically embedded circles). Let $\lambda: \Omega \rightarrow \mathbf{C}$ be a measurable function with $\|\lambda\|_{\infty}<1$. Then a solution to the Beltrami equation consists of a closed Riemann surface $N$ (homeomorphic to $M$ ) and a quasiconformal map $f$ from $\Omega$ into $N$ such that $\partial_{\bar{z}} f(z) / \partial_{z} f(z)=\lambda(z)$ a.e. $z \in \Omega$. Our methods may be extended to construct approximations to solutions of the Beltrami equation in this case by using the Circle Packing Theorem on arbitrary surfaces [T, Theorem 13.7.1 and Corollary 13.6.2] which implies that for any closed oriented surface $S$ and any triangulation on $S$, there is a metric of constant curvature on $S$, uniquely determined up to a scalar multiple, and a circle packing $P$, unique up to a conformal transformation of $S$, whose nerve is isotopic to the 1-skeleton of the triangulation. Note that a metric of constant curvature on $S$ determines a unique conformal structure on $S$ and, conversely, a conformal structure (=complex structure) on $S$ determines a metric of constant curvature on $S$, unique up to a scalar multiple.

The construction of the approximations is briefly sketched as follows. For any small $\delta>0$, construct a $P L$ complex $\left(\bar{S}_{\delta}, \bar{s}_{\delta}\right)$ whose 1-simplexes are all geodesic arcs in $N$ of length between $c_{1} \delta$ and $C_{1} \delta$ for some $c_{1}>0, C_{1}>c_{1}$ independent of $\delta$. Let $\left(S_{\delta}, s_{\delta}\right)$ be a $P L$ subcomplex of $\left(\bar{S}_{\delta}, \bar{s}_{\delta}\right)$ such that: (i) $\bar{\Omega}_{\delta} \equiv s_{\delta}\left(\left|S_{\delta}\right|\right)=\bar{s}_{\delta}\left(\left|S_{\delta}\right|\right)$ contains $\Omega$, and (ii) $\bar{\Omega}_{\delta}$ is a 2-manifold whose boundary $\partial \bar{\Omega}_{\delta}$ is a finite union of Jordan curves $J_{\delta}^{1}, \ldots, J_{\delta}^{j}$, such that $J_{\delta}^{i}$ corresponds to a unique Jordan curve $J^{i}$ of $\partial \Omega_{\delta}$ and vice versa, and the 
distance of $J_{\delta}^{i}$ with $J^{i}$ is convergent to 0 when $\delta \rightarrow 0$. Let $\left(\widetilde{S}_{\delta}, \tilde{s}_{\delta}\right)$ be a $P L$ subcomplex of $\left(S_{\delta}, s_{\delta}\right)$ with the property that $\widetilde{\Omega}_{\delta} \equiv \tilde{s}_{\delta}\left(\left|\tilde{S}_{\delta}\right|\right)$ is a 2-submanifold (with boundary) contained in $\Omega$ and $\widetilde{\Omega}_{\delta}$ is a union of annular regions. Any triangle $s_{\delta}(\sigma)$ of $\left(S_{\delta}, s_{\delta}\right) \quad\left(\sigma \in S_{\delta}\right)$ is isometric to a triangle in $S^{2}, \mathbf{R}^{2}$, or $H^{2}$ whose edges have lengths between $c_{1} \delta$ and $C_{1} \delta$, and hence $\left(1+\varepsilon_{\delta}\right)$ quasiconformal homeomorphic to the triangle $\Delta(\sigma)$ in $\mathbf{R}^{2}$ with the same edge lengths by some map $v_{\sigma}$ which is isometric on 1-simplexes, where $\varepsilon_{\delta} \rightarrow 0$. Let

$$
\lambda\left(s_{\delta}(\sigma)\right)= \begin{cases}\frac{1}{A\left(s_{\delta}(\sigma)\right)} \iint_{s_{\delta}(\sigma)} \lambda(z) d A, & \text { if } s_{\delta}(\sigma) \subset \widetilde{\Omega}_{\delta}, \\ 0, & \text { otherwise, }\end{cases}
$$

and define $\lambda_{\delta}: \Omega \rightarrow \mathbf{C}$ by $\lambda_{\delta}(z)=\lambda\left(s_{\delta}(\sigma)\right)$ a.e. $z \in s_{\delta}(\sigma) \cap \Omega$. Then $\lambda_{\delta}(z) \rightarrow$ $\lambda(z)$ a.e. $z \in \Omega$.

Let $\sigma \in \widetilde{S}_{\delta}$, and let $\psi_{\sigma}: \mathbf{C} \rightarrow \mathbf{C}$ be defined by (3.4) where $\lambda(Q)$ is replaced by $\lambda\left(s_{\delta}(\sigma)\right)$. Let $n=2[1 / 2 \delta]$, and let $H_{\delta / n}$ be the simplicial complex constructed in $\S 3$. Let $H\left(\Delta^{\prime}(\sigma)\right)$ be the collection of all triangles of $H_{\delta / n}$ lying in $\Delta^{\prime}(\sigma) \equiv$ $\psi_{\sigma}(\Delta(\sigma))$ and having distance at least $\delta / 2 n$ from $\partial \psi_{\sigma}(\Delta(\sigma))$. Then $H\left(\Delta^{\prime}(\sigma)\right)$ is a simplicial complex and $\Delta_{i}^{\prime}(\sigma)=\left|H\left(\Delta^{\prime}(\sigma)\right)\right|$ is some polyhedral Jordan domain in $\Delta^{\prime}(\sigma)$.

Let $\left(L_{n}, l_{n}\right)$ be a $P L$ complex on the boundary of $\Delta(\sigma)$ such that any edge of $\Delta(\sigma)$ is a union of $n 1$-simplexes of the same length. Then we can construct a $C$-quasiconformal $P L$ complex $\left(H_{b}\left(\Delta^{\prime}(\sigma)\right), h_{b}\right)$ on $\overline{\Delta^{\prime}(\sigma) \backslash \Delta_{i}^{\prime}(\sigma)}$ such that $\left(H_{b}\left(\Delta^{\prime}(\sigma)\right), h_{b}\right) / \partial \Delta^{\prime}(\sigma)=\left(L_{n}, \psi_{\sigma} \circ l_{n}\right)$ and $\left(H_{b}\left(\Delta^{\prime}(\sigma)\right), h_{b}\right) / \partial \Delta_{i}^{\prime}(\sigma)=$ $\left(H\left(\Delta^{\prime}(\sigma)\right)\right.$, id $) / \partial \Delta_{i}^{\prime}(\sigma)$. Then $\left(H_{b}\left(\Delta^{\prime}(\sigma)\right), h_{b}\right)$ and $\left(H\left(\Delta^{\prime}(\sigma)\right)\right.$, id $)$ fit together and give a $C$-quasiconformal $P L$ complex $\left(T\left(\Delta^{\prime}(\sigma)\right), t^{\prime}\right)$ on $\Delta^{\prime}(\sigma)$. It follows that $\left(T(\sigma) \equiv T\left(\Delta^{\prime}(\sigma)\right), t_{\sigma} \equiv v_{\sigma}^{-1} \circ \psi_{\sigma}^{-1} \circ t^{\prime}\right)$ is a $C$-quasiconformal $P L$ complex on $s_{\delta}(\sigma)=v_{\sigma}^{-1}(\Delta(\sigma))$ and is equal to $\left(L_{n}, v_{\delta}^{-1} \circ l_{n}\right)$ on $\partial s_{\delta}(\sigma)$. These $P L$ complexes yield a $C$-quasiconformal $P L$ complex $\left(\widetilde{T}_{\delta}, \tilde{t}_{\delta}\right)$ for $\widetilde{\Omega}=$ $\bigcup_{\sigma \in \widetilde{S}_{\delta}} s_{\delta}(\sigma)$. For the union of annular regions $\bar{\Omega} \backslash$ int $\widetilde{\Omega}_{\delta}$, it is easy to find some $C$-quasiconformal $P L$ complex on it which is equal to $\left(\widetilde{T}_{\delta}, \tilde{t}_{\delta}\right) / \partial \widetilde{\Omega}_{\delta}$ on $\partial \widetilde{\Omega}_{\delta}$, and any vertex in $\bar{\Omega}_{\delta} \backslash \widetilde{\Omega}_{\delta}$ is the end of at most six 1-simplexes and any 1 simplex has length bounded by $C \delta / n$. This $P L$ complex together with $\left(\widetilde{T}_{\delta}, \tilde{t}_{\delta}\right)$ gives a $P L$ complex $\left(T_{\delta}, t_{\delta}\right)$ on $\bar{\Omega}_{\delta}$. Now Lemma 3.1 holds with some obvious changes.

Let $B_{\delta}^{i}, i=1, \ldots, j$, be the simplicial subcomplex of $T_{\delta}$ such that $\left|B_{\delta}^{i}\right|=$ $t_{\delta}^{-1}\left(J_{\delta}^{i}\right)$. Set $\bar{T}_{\delta}=T_{\delta} \cup \bigcup_{i=1}^{j}\left(B_{\delta}^{i} * V_{i}\right)$, where $V_{i} \notin T_{\delta}$ are some added vertices. Then $\bar{T}_{\delta}$ is a closed oriented 2-manifold homeomorphic to $M$ by some sense preserving homeomorphism extending $t_{\delta}:\left|T_{\delta}\right| \subset\left|\bar{T}_{\delta}\right| \rightarrow \bar{\Omega}_{\delta} \subset M$. Using the Circle Packing Theorem of [T, Theorem 13.7.1 and Corollary 13.6.2] mentioned above, there is a unique Riemann surface $N_{\delta}$ (homeomorphic to $M$ ), and a circle packing $P_{\delta}$ on $N_{\delta}$, unique up to a conformal transformation of $N_{\delta}$, 
whose nerve is isotopic to the 1 -skeleton of $\bar{T}_{\delta}$. Then there is a map $g_{\delta}:\left|T_{\delta}\right| \rightarrow$ $N_{\delta}$ which sends each vertex of $T_{\delta}$ to the center of the corresponding disk of $P_{\delta}$, and each 1 -simplex linearly to the shortest geodesic joining the corresponding disk of $P_{\delta}$, and each 1-simplex linearly to the shortest geodesic joining the corresponding centers of disk, such that $g_{\delta}$ restricted to any triangle $\sigma \in T_{\delta}$ is the map which admits minimal conformal distortion, where the conformal structure of $\sigma$ is chosen in such a way that $\sigma$ is linearly conformal equivalent to any equilateral triangle in $\mathbf{C}$.

Define $f_{\delta}: \Omega \rightarrow N_{\delta}$ to be the restriction of $g_{\delta} \circ t_{\delta}^{-1}$. If $\left|\bar{T}_{\delta}\right| \cong S^{2}$, we may assume that $f_{\delta}$ is fixed at some prescribed point. It is obvious that the Ring Lemma, the Hexagonal Lemma, and the Length-Area Lemma for circle packings in arbitrary surface also hold. So by the same arguments as in $\S 4$, one may prove that $g_{\delta} \circ t_{\delta}^{-1}$ and hence $f_{\delta}$ are $C$-quasiconformal, and

$$
\lim _{\delta \rightarrow 0} \frac{\partial_{z} f_{\delta}(z)}{\partial_{z} f_{\delta}(z)}=\lambda(z) \quad \text { a.e. } z \in \Omega \text {. }
$$

From the construction, $\bar{\Omega}_{\delta} \supseteq \Omega$ converges to $\Omega$ as $\delta \rightarrow 0$ and the inclusions $\Omega \subset \bar{\Omega}_{\delta}$ are homotopy equivalences. So any two of the manifolds (with conformal structure) $D_{\delta} \equiv g_{\delta} \circ t_{\delta}^{-1}\left(\Omega_{\delta}\right) \subset N_{\delta}$ are $C$-quasiconformally homeomorphic. Then, for any sequence $\delta_{k} \rightarrow 0$ there are subsequences, still denoted by $\delta_{k}$, such that $D_{\delta_{k}}$ converges (in the Teichmüller space of the underlying 2-manifold) to some open 2-manifold $D$ with conformal structure, and $f_{\delta_{k}}$ converges to some quasiconformal map $f: \Omega \rightarrow D$ with complex dilatation $\lambda$. By the Length-Area Lemma, $D_{\delta_{k}}$ becomes closer and closer as $k \rightarrow \infty$ to the complement in the closed manifold $N_{\delta_{k}}$ of the union of disjoint closed round disks of $P_{\delta_{k}}$ corresponding to the vertices $P_{i}, i=1,2, \ldots, j$. One can then easily show that $N_{\delta_{k}}$ are $C$-quasiconformal homeomorphisms between $D_{\delta_{k}}$ when restricting on $D_{\delta_{k}}$. Therefore, we may choose some subsequence of $N_{\delta_{k}}$ converging to some closed 2-manifold $N$ with conformal structure. Hence, $D$ is an open submanifold of $N$ which can be expressed as the complement of some union $R$ of $j$ disjoint closed round disks. Then we have

Theorem 5.1. Let $\Omega$ be the interior of a closed domain $\bar{\Omega}$ in a closed 2manifold $M$ with conformal structure such that $\partial \bar{\Omega}$ consists of a finite number (possibly empty) of Jordan curves. Let $\lambda: \Omega \rightarrow \mathbf{C}$ be a measurable function with $\|\lambda\|_{\infty}<1$. Then there exist some closed 2-manifold $N$ with conformal structure, homeomorphic to $M$; a finite union $R$ of disjoint closed round disks in $N$; and a quasiconformal homeomorphism $f: \Omega \rightarrow N \backslash R$ such that $f$ has complex dilatation $\lambda(z)$ for a.e. $z \in \Omega$.

As a corollary, any finitely connected Riemann surface contained in $M$ is conformal equivalent to some closed Riemann surface with some round disks or points removed. This extends a result of Köebe, which states that a finitely connected planar region is conformal equivalent (in an essentially unique way) 
to a region bounded by round circles and points (see, e.g., [G, pp. 201-217]). This corollary can also be be proved by lifting $M$ to the universal cover (which is the unit disk), and applying an extended version of Köebe's result for the preimage of $\Omega$ under the covering map.

\section{REFERENCES}

[A] L. Ahlfors, Lectures on quasiconformal mappings, Van Nostrand, Princeton, N. J., 1966.

[AB] L. Ahlfors and L. Bers, Riemann's mapping theorem for variable metrics, Ann. of Math. 72 (1960), 385-404.

[Be] L. Bers, Riemann surfaces (mimeographed lectures notes), New York University, 1957-58.

[BN] L. Bers and L. Nirenberg, On a representation theorem for linear elliptic systems with discontinuous coefficients and its applications, Convegno Intern. Equazioni Lineari all Derivate Partiali, Rome, 1954, pp. 111-140.

[Bo] B. V. Boyarskii, Generalized solutions of systems of differential equations of first order and elliptic type with discontinuous coefficients, Mat. Sb. (N. S.) 43 (85) (1957), 451-503 (Russian).

[C] S. S. Chern, An elementary proof of the existence of isothermal parameters on a surface, Proc. Amer. Math. Soc. 6 (1955), 781-782.

[G] G. M. Golusin, Geometrische Funktionentheorie, VEB Deutsche Verlag der Wiss., Berlin, 1957.

[H] Z.-X. He, An estimate for hexagonal circle packings, J. Differential Geom. 33 (1991).

[K] A. Korn, Zwei Anwendungen der Methode der sukzessiven Annäherungen, Schwarz Festschrift, Berlin, 1919, pp. 215-229.

[La] M. A. Lavrentiev, Sur une class des représentations continues, Mat. Sb. (N. S.) 42 (1935), 407-434.

[LV] O. Lehto and K. I. Virtanen, Quasiconformal mappings in the plane, Springer-Verlag, New York, 1973.

[Li] L. Lichtenstein, Zur Theorie der konformen Abbildungen; Konforme Abbildungen nichtanalytischer singularitätenfreir Flächenstücke auf ebene Gebiete, Bull. Acad. Sci. Cracovie (1916), 192-217.

[MR] A. Marden and B. Rodin, On Thurston's formulation and proof of Andreev's theorem, preprint.

[Ma] O. Martio, Boundary values and injectiveness of the solutions of Beltrami equations, Ann. Acad. Sci. Fenn. A I Math. 402 (1967).

[Mo] C. B. Morrey, On the solution of quasilinear elliptic partial differential equations, Trans. Amer. Math. Soc. 43 (1938), 126-166.

[RS] B. Rodin and D. Sullivan, The convergence of circle packing to the Riemann mapping, J. Differential Geom. 26 (1987), 349-360.

[T] W. Thurston, The geometry and topology of 3-manifolds, Princeton University Notes, Princeten, N. J., 1979.

Department of Mathematics, University of California, San Diego, la Jolla, CALIFORNia 92093

Current address: Department of Mathematics, Princeton University, Princeton, New Jersey 08544 